\title{
Equilibration via Gaussification in Fermionic Lattice Systems
}

\author{
M. Gluza, ${ }^{1}$ C. Krumnow, ${ }^{1}$ M. Friesdorf, ${ }^{1}$ C. Gogolin, ${ }^{2,3}$ and J. Eisert ${ }^{1}$ \\ ${ }^{1}$ Dahlem Center for Complex Quantum Systems, Freie Universität Berlin, 14195 Berlin, Germany \\ ${ }^{2}$ ICFO-Institut de Ciencies Fotoniques, Mediterranean Technology Park, 08860 Castelldefels (Barcelona), Spain \\ ${ }^{3}$ Max-Planck-Institut für Quantenoptik, Hans-Kopfermann-Straße 1, 85748 Garching, Germany
}

(Received 19 February 2016; published 4 November 2016)

In this Letter, we present a result on the nonequilibrium dynamics causing equilibration and Gaussification of quadratic noninteracting fermionic Hamiltonians. Specifically, based on two basic assumptions-clustering of correlations in the initial state and the Hamiltonian exhibiting delocalizing transport-we prove that non-Gaussian initial states become locally indistinguishable from fermionic Gaussian states after a short and well controlled time. This relaxation dynamics is governed by a power-law independent of the system size. Our argument is general enough to allow for pure and mixed initial states, including thermal and ground states of interacting Hamiltonians on large classes of lattices as well as certain spin systems. The argument gives rise to rigorously proven instances of a convergence to a generalized Gibbs ensemble. Our results allow us to develop an intuition of equilibration that is expected to be more generally valid and relates to current experiments of cold atoms in optical lattices.

DOI: 10.1103/PhysRevLett.117.190602

Despite the great complexity of quantum many-body systems out of equilibrium, local expectation values in such systems show the remarkable tendency to equilibrate to stationary values that do not depend on the microscopic details of the initial state, but rather can be described with few parameters using thermal states or generalized Gibbs ensembles [1-3]. Such behavior has been successfully studied in many settings theoretically and experimentally, most notably in instances of quantum simulations in optical lattices $[2,4,5]$.

By now, it is clear that, despite the unitary nature of quantum mechanical evolution, local expectation values equilibrate due to a dephasing between the eigenstates [3,6-12]. So far it is, however, unclear why this dephasing tends to happen so rapidly. In fact, experiments often observe equilibration after very short times, which are independent of the system size [5,13], while even the best theoretical bounds for general initial states of concrete systems diverge exponentially $[2,12]$. This discrepancy poses the challenge of precisely identifying the equilibration time, which constitutes one of the main open questions in the field [1-3].

What is more, only little is known about how exactly the equilibrium expectation values emerge. Because of the exponentially many constants of motion present in quantum many-body systems, corresponding to the overlaps with the eigenvectors of the system, there seems to be no obvious reason why equilibrium values often only depend on a few macroscopic properties such as temperature or particle number. In short: it is unclear how precisely the memory of the initial conditions is lost during time evolution.

To make progress towards a solution of these two problems, it is instructive to study the behavior of noninteracting particles captured by so-called quadratic or free models. In these models, the time evolution of so-called Gaussian states, which are fully described by their correlation matrix, is particularly simple to describe. While studying the time evolution of such states provides valuable insight into the spreading of particles and equilibration, it is unclear if and under which conditions general non-Gaussian initial states out of equilibrium end up appearing Gaussian.

In this Letter, we address this question: we show under which conditions very general non-Gaussian initial states become locally indistinguishable from Gaussian states with the same second moments. This mechanism is much reminiscent of actual thermalization, in that an initially complex setting appears to converge to a high-entropy state that is defined by astoundingly few parameters only. In this way, we present a significant step forward in the theory of equilibration of quantum many-body systems that have been pushed out of equilibrium. Furthermore, our work suggests that for quadratic models, Gaussification can be seen as a genuine mechanism of nonequilibrium dynamics, complementing and playing a significant role in equilibration.

Our results hold for a remarkably large class of initial states, gapped interacting models evolving, after a so-called quench, in time under a quadratic fermionic Hamiltonian with finite ranged interactions. This family of Hamiltonians notably includes the case of noninteracting ultracold fermions realizable in optical lattices. By virtue of the Jordan-Wigner transformation, our results also apply to certain spin models. We formulate our results in the form of a rigorously proven theorem, which at the same time provides an intuitive explanation of the physics behind our result. We find Gaussification to be a consequence of two natural assumptions, namely exponential clustering of correlations in the initial state and what we call delocalizing transport. 
Setting.-We begin by precisely stating the physical setting that we consider. For notational convenience, let $\mathcal{L}$ be a $d_{\mathcal{L}}$-dimensional cubic lattice with $V$ lattice sites. Each site $r \in \mathcal{L}$ is associated with a fermionic orbital with fermionic creation and annihilation operators $f_{r}^{\dagger}$ and $f_{r}$. We collect them in a vector $c=\left(f_{1}, f_{1}^{\dagger}, \ldots, f_{V}, f_{V}^{\dagger}\right)$. All results can be generalized to fermions with internal degrees of freedom on Kagomé, honeycomb, or other geometries. The Hamiltonian of a quadratic fermionic system is then of the form

$$
H=\sum_{j, k=1}^{2 V} c_{j}^{\dagger} h_{j, k} c_{k}
$$

with Hermitian coupling matrix $h$. The time evolution of annihilation operators in the Heisenberg picture under such a Hamiltonian is given by

$$
c_{j}(t):=e^{i H t} c_{j} e^{-i H t}=\sum_{k=1}^{2 V} W_{j, k}(t) c_{k}
$$

with the propagator $W(t):=e^{-2 i t h}$.

Next, we introduce the concept of Gaussian states and Gaussification. Define the correlation matrix $\gamma$ of a state $\rho$ as the matrix of its second moments, i.e., $\gamma_{j, k}:=\operatorname{tr}\left(\rho c_{j}^{\dagger} c_{k}\right)$. A convenient characterization of Gaussian states is the following: they are the states that maximize the von Neumann entropy given the expectation values collected in the correlation matrix. For every state $\rho$, we hence define its Gaussified version $\rho_{G}$ as the Gaussian state with the correlation matrix of $\rho$, i.e., $\operatorname{tr}\left(\rho_{G} c_{j}^{\dagger} c_{k}\right)=\operatorname{tr}\left(\rho c_{j}^{\dagger} c_{k}\right)$.

Assumptions.-Our main theorem holds for initial states (including non-Gaussian ones) with a form of decay of correlations that evolve under quadratic Hamiltonians that exhibit a form of transport that we define below. We now make these two conditions precise, starting with the correlation decay:

Definition 1.-(Exponential clustering of correlations) We say that a state $\rho$ exhibits exponential clustering of correlations with length scale $\xi>0$ and constant $C_{\text {Clust }}>0$ if, for any two operators $A, B$ with $\|A\|=\|B\|=1$, we have

$$
\begin{aligned}
& |\operatorname{tr}(\rho A B)-\operatorname{tr}(A \rho) \operatorname{tr}(B \rho)| \\
& \quad \leq C_{\text {Clust }}|\operatorname{supp}(A)||\operatorname{supp}(B)| e^{-d(A, B) / \xi} .
\end{aligned}
$$

Here $d(A, B)$ is taken to be the natural distance on the lattice between the supports $\operatorname{supp}(A), \operatorname{supp}(B)$ of $A$ and $B$ and $\|\cdot\|$ denotes the operator norm.

Ground states of interacting gapped local Hamiltonians $[14,15]$ as well as thermal states of arbitrary nonquadratic fermionic systems [16] at sufficiently high temperature have exponential clustering of correlations as defined in Definition 1. Thus, the initial state could be prepared within a quench scenario where the Hamiltonian is changed from a gapped interacting model to a quadratic Hamiltonian which
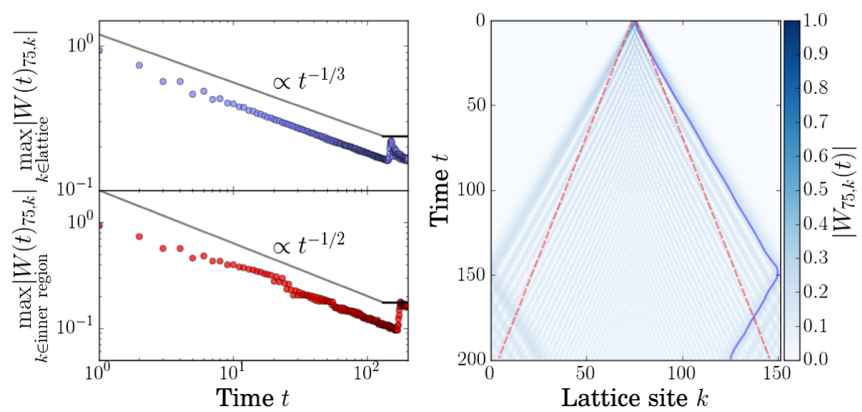

FIG. 1. The right panel shows a numerical study of the spreading of the support of a fermionic annihilation operator described in Eq. (2) under the evolution of the quadratic hopping Hamiltonian $H=-\sum_{j}\left(f_{j}^{\dagger} f_{j+1}+f_{j+1}^{\dagger} f_{j}\right)$ on a one-dimensional chain of 150 sites with periodic boundary conditions. The support expands ballistically, creating the Lieb-Robinson cone. The left panel shows the suppression of different elements of the propagator in time. The plot at the top shows the evolution of the maximum taken over the full lattice $\max _{k}\left|W_{75, k}(t)\right|$, where in the lower plot the maximum inside the inner region of the Lieb-Robinson cone (between the red dashed lines) is plotted. The maximum taken over the full lattice is reached for $k$ in the wave front (indicated by the blue curve in the right panel) and the suppression goes as $t^{-1 / 3}$, while in the bulk of the cone, the suppression is proportional to $t^{-1 / 2}$. The suppression stabilizes, once the wave fronts collide.

governs the nonequilibrium dynamics. To reemphasize, by no means is the initial state assumed to be in any specific relation to properties of the latter quadratic Hamiltonian.

For our proof of local relaxation towards a Gaussian state, we further assume that the quadratic Hamiltonian exhibits transport in the following sense:

Definition 2.-(Delocalizing transport) A quadratic Hamiltonian with propagator $W$ on a $d_{\mathcal{L}}$-dimensional cubic lattice of volume $V$ exhibits delocalizing transport with constants $C_{\text {Trans }}>0, \alpha_{\text {Trans }}>d_{\mathcal{L}} / 4$ and recurrence time $t_{\text {Rec }}>0$ if, for all $t \in\left(0, t_{\text {Rec }}\right]$, we have that

$$
\forall j, k:\left|W_{j, k}(t)\right| \leq C_{\text {Trans }} \max \left\{t^{-\alpha_{\text {Trans }}}, V^{-\alpha_{\text {Trans }}}\right\} .
$$

The intuition behind this definition is that an initially localized fermionic operator will spread over a large area, such that its component on a single localized operator is dynamically suppressed. In particular, such a suppression with $\alpha_{\text {Trans }}=d_{\mathcal{L}} / 3$ can be proven for quadratic hopping Hamiltonians (see the Supplemental Material [17] and also Fig. 1) and the critical Ising model. In finite dimensional systems, any nontrivial bound of the form (4) is eventually violated due to the recurrent nature of their dynamics. For quadratic hopping Hamiltonians, it can be shown that the recurrence time grows at least like $V^{6 / 7 d_{\mathcal{L}}}$ with the system size.

Main result.-Our main result can be stated as follows:

Theorem 1.-(Gaussification in finite time) Consider a family of systems on cubic lattices of increasing volume $V$. 
Let the initial states exhibit exponential clustering of correlations, and let the Hamiltonians be quadratic finite range and have delocalizing transport with the corresponding constants $\xi, C_{\text {Clust }}, C_{\text {Trans }}, \alpha_{\text {Trans }}$ independent of $V$. Then for any local operator $A$ on a fixed finite region and any $0<\nu<4 \alpha_{\text {Trans }}-d_{\mathcal{L}}$, there is a $V$ independent constant $C_{\text {Total }}$ such that for any $t \leq \min \left(t_{\operatorname{Rec}}, V\right)$

$$
\left|\operatorname{tr}[A(t) \rho]-\operatorname{tr}\left[A(t) \rho_{G}\right]\right| \leq C_{\text {Total }} t^{-4 \alpha_{\text {Trans }}+d_{\mathcal{L}}+\nu} .
$$

Consequently, if the recurrence time $t_{\operatorname{Rec}}$ increases unboundedly as some function of $V$, then, given an error $\epsilon>0$, there exists a relaxation time $t_{\text {Relax }}>0$ independent of the system size such that for all times $t \in\left[t_{\text {Relax }}, t_{\text {Rec }}\right]$ it holds that $\left|\operatorname{tr}[A(t) \rho]-\operatorname{tr}\left[A(t) \rho_{G}\right]\right| \leq \epsilon$.

The theorem states that for all times in the interval $\left[t_{\text {Relax }}, t_{\text {Rec }}\right]$ the expectation value of any local observable in the time evolved state $\rho(t)$ will agree up to an error $\epsilon$ with the expectation value in the Gaussian state $\rho_{G}(t)$, which has the same second moments as $\rho(t)$. With this, we find that the expectation values of all local observables can be approximated by a decomposition according to Wick's theorem and that it will be impossible to distinguish the true state $\rho$ from the fermionic Gaussian state $\rho_{G}$ by any local measurement on a fixed finite local region $S$. Note that since $t_{\text {Relax }}$ is independent of the system size, but $t_{\text {Rec }}$ increases with its volume, for any arbitrarily small $\epsilon$, there always exists a system size such that $t_{\text {Rec }}>t_{\text {Relax }}$, and the interval where the theorem applies grows as a function of the system size.

We compare our general, rigorous, analytical result with a numerical simulation in Fig. 2 that shows an experimentally detectable signature of the Gaussification of a density-density correlator. The comparison reveals that our bound correctly reproduces the physical behavior in the sense that the true Gaussification dynamics follows a power-law. What is not correctly reproduced is the exponent of that power law, but we understand where the discrepancy between the observed $t^{-1}$ decay and the $t^{-1 / 3+\nu}$ (for arbitrarily small $\nu$ ) bound for $\alpha_{\text {Trans }}=1 / 3$ originates from: the reason is that the provable decay with $\alpha_{\text {Trans }}=$ $1 / 3$ for the considered model roots in the slow decay of the matrix elements of the propagator at the wave front of the Lieb-Robinson cone. The elements in the bulk of the LiebRobinson cone can numerically be found to be suppressed as $t^{-1 / 2}$ leading to an effective $\alpha_{\text {Trans }}=1 / 2$ for the vast majority of matrix elements (see Fig. 1). Assuming this effective $\alpha_{\text {Trans }}=1 / 2$ in Theorem 1 leads to a suppression with $t^{-1}$.

The key steps in the proof, are based on three main physical ingredients: finite speed of propagation in lattice systems, homogeneous suppression of matrix elements of the propagator due to delocalizing transport, and exponential clustering of correlations in the initial state. The full proof with all details of the involved combinatorics can be found in the Supplemental Material [17].

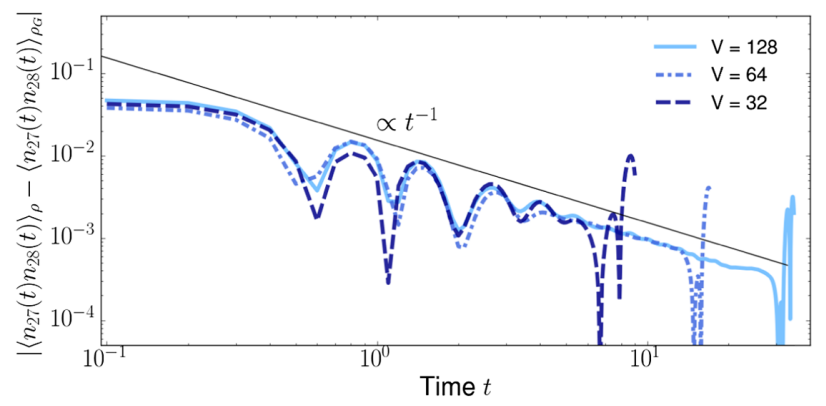

FIG. 2. Numerical study of the evolution of a nearest-neighbor density-density correlator for system sizes $V=32,64,128$ under the quadratic hopping Hamiltonian $H=-\sum_{j}^{V}\left(f_{j}^{\dagger} f_{j+1}+\right.$ $\left.f_{j+1}^{\dagger} f_{j}\right)$. The initial states are the ground states of the interacting spinless Fermi-Hubbard model $H_{\mathrm{FH}}=H+U \sum_{j}^{V} n_{j} n_{j+1}+$ $\sum_{j} \omega_{j} n_{j}$ with $U=2$, and weak on site disorder $w_{j}$ drawn independently from a Gaussian distribution with variance $1 / 4$. For consistency, we have initially drawn 128 random numbers $w_{j}$ and used the first $V$ of them for different system sizes. All calculations were performed with periodic boundary conditions at half filling. The difference between the expectation values in the state $\rho$ and its Gaussified version $\rho_{G}$ of the density-density correlator between sites 27 and 28 as a function of time is suppressed approximately like $t^{-1}$, as indicated by the black line. At late times, due to the finite size of the system, recurrences occur, leading to an increase of the difference. Increasing the system size only shifts the recurrence time $t_{\text {Rec }}$ but leaves the decay behavior unchanged. The visible oscillations depend on details of the model and initial state. The time evolution was performed by using Eq. (2).

Proof.-We expand the local operator $A$ supported in a fixed finite region $S$ in the basis of fermionic operators. To that end, let $\tilde{S}:=\left\{s_{r}\right\}$ for $r \in[2|S|]:=\{1, \ldots, 2|S|\}$ be the set of indices of all elements of the vector $c$ with support in $S$, then

$$
A(t)=\sum_{b_{1}, \ldots, b_{2|S|}=0}^{1} a_{b_{1}, \ldots, b_{2|S|}} c_{S_{1}}(t)^{b_{1}} \ldots c_{S_{2|S|}}(t)^{b_{2|S|}} .
$$

Without loss of generality, we assume $\|A\|=1$, such that $\left|a_{b_{1}, \ldots, b_{2 m}}\right| \leq 1$. Thus,

$$
\begin{aligned}
& \left|\operatorname{tr}[A \rho(t)]-\operatorname{tr}\left[A \rho_{G}(t)\right]\right| \\
& \quad \leq 2^{2|S|} \max _{J \subset \tilde{S}}\left|\sum_{\left(k_{j}\right)_{j \in J} \in[2 V]^{\times|J|}} \operatorname{tr}\left[\prod_{j \in J} W_{j, k_{j}}(t) c_{k_{j}}\left(\rho-\rho_{G}\right)\right]\right| .
\end{aligned}
$$

Here and in the following, all products are meant to be performed in increasing order.

We assumed that the Hamiltonian has finite range interactions; i.e., there exists a fixed length $l_{0}$, such that $h_{j, k}=0$ whenever $d(j, k)>l_{0}$, where $d(j, k):=d\left(c_{j}, c_{k}\right)$. Such models satisfy Lieb-Robinson bounds [22], which in our setting can be stated as follows: 
Lemma.-(Lieb-Robinson bound for quadratic systems [23]) For any quadratic fermionic Hamiltonians $H$ with finite range interactions there exist constants $C_{\mathrm{LR}}, \mu, v>0$ independent of the system size, such that its propagator $W$ fulfills the bound

$$
\left|W_{j, k}(t)\right| \leq C_{\mathrm{LR}} e^{\mu[v|t|-d(j, k)]} .
$$

The Lieb-Robinson bound tells us that $c_{j}(t)$ and $c_{k}(t)$ essentially still have disjoint support as long as $t$ is small enough such that $v|t| \ll d(j, k)$. We can hence restrict the sum in Eq. (7) to those $k_{j}$ whose $\min _{s \in \tilde{S}} \mathrm{~d}\left(k_{j}, s\right)$ is smaller than $\left(v+2 v_{\epsilon}\right)|t|$ for some fixed $v_{\epsilon}>0$. The total contribution of the neglected terms can be bounded explicitly and, importantly, is independent of $V$ and exponentially suppressed in $\left|v_{\epsilon} t\right|$.

For each of the remaining summands in Eq. (7), it is now important to keep track of the distribution of the indices $k_{j}$ inside the cone. For this purpose, we define the $\Delta$ partition $P_{\Delta}$ of a subindex set $J \subset \tilde{S}$ and sequence of indices $\left(k_{j}\right)_{j_{\in} J}$ as the unique decomposition of $J$ into subsets (patches) $p$ in the following way: the patches are constructed such that for any two subindices within any given patch $p$ there is a connecting chain of elements from that patch in the sense that the distance between two consecutive $c_{k_{j}}$ with $j \in p$ along that chain is not greater than $\Delta$ and the distance between any two $c_{k_{j}}, c_{k_{j}^{\prime}}$ with $j, j^{\prime}$ from different patches is larger than $\Delta$. For each patch $p$ in the $\Delta$ partition of a given summand in Eq. (7), we define a corresponding operator

$$
\hat{P}_{p}^{\left(k_{j}\right)_{j \in p}}:=\prod_{j \in p} W_{j, k_{j}}(t) c_{k_{j}} .
$$

We can then reorder the factors in Eq. (7) to write the product as a product over these operators. The exponential clustering of correlations (Definition 1) in the initial state allows us to factor the patches if we scale $\Delta$ suitably with $|t|$. Concretely, for $\sigma \in\left\{\rho, \rho_{G}\right\}$, the expectation values appearing in Eq. (7), which we denote by $\langle\cdot\rangle_{\sigma}$, can be approximated as follows:

$$
\left\langle\prod_{p \in P_{\Delta}} \hat{P}_{p}^{\left(k_{j}\right)_{j \in p}}\right\rangle_{\sigma} \approx \prod_{p \in P_{\Delta}}\left\langle\hat{P}_{p}^{\left(k_{j}\right)_{j \in p}}\right\rangle_{\sigma} .
$$

The error thereby introduced is exponentially suppressed with the ratio of patch distance to correlation length $\Delta / \xi$.

It remains to bound the contribution from the factorized patches that are completely inside the Lieb-Robinson cone. Note that the right-hand side of Eq. (10) is nonzero only if all the patches are of even size, as $\rho$ and $\rho_{G}$ have an even particle number parity. Moreover, as the second moments of $\rho$ and $\rho_{G}$ are equal, the difference of the right-hand side for $\sigma=\rho$ and $\sigma=\rho_{G}$ vanishes whenever all patches have size 2 . Hence, only partitions that contain at least one patch of size at least 4 contribute. The delocalizing transport of the Hamiltonian implies that the contribution from such larger patches however is dynamically suppressed. Whenever $|p| \geq 4$, it holds that

$$
\left|\left\langle\hat{P}_{p}^{\left(k_{j}\right)_{j \in p}}\right\rangle_{\sigma}\right| \leq C_{\text {Trans }}^{4} t^{-4 \alpha_{\text {Trans }}}
$$

as long as $t$ is small enough given $V$. The influence of possible patches of size 2 in the same decomposition makes it necessary to bound the overall contribution with an involved recursive and combinatorial argument. However, effectively the dynamical suppression in Eq. (11) allows us to derive a bound that increases with the patch size $\Delta$, as the number of possible patch-configurations grows with $\Delta$, but is algebraically suppressed in time $t$. Choosing $\Delta=\max \left(1, t^{\nu / 4 d_{\mathcal{L}}}\right)$ for some $0<\nu<4 \alpha_{\text {Trans }}-d_{\mathcal{L}}$, we obtain an at least algebraic suppression with $t$ of all terms and thereby of the difference $\left|\operatorname{tr}(A(t) \rho)-\operatorname{tr}\left(A(t) \rho_{G}\right)\right|$.

Physical implications and applications.-The Gaussification result presented above also has profound implications for the study of equilibration of quantum many-body systems. Whenever the second moments equilibrate, which is often observed [5,24-29], our results imply that the full reduced density matrix becomes stationary. The numerical study presented in Fig. 2 shows that the power law appearing in Theorem 1 is not an artifact of our proof strategy but reflects the underlying physics, that can moreover be observed in experiments. The quadratic models considered here constitute a "theoretical laboratory", in which the mechanisms of Gaussification and equilibration can be very precisely and quantitatively characterized, and all specifics of the processes laid out. This does not mean that the physics we address is very specific to quadratic Hamiltonians: we expect the fundamental mechanisms underlying the result-local relaxation due to transport and initial clustering of correlations-to be, the reason for relaxation in a wide classes of interacting models [30] and also classical ones [31]. The intuition, reminiscent of a quantum central limit theorem [32], that incommensurate influences of further and further separated regions lead to mixing and relaxation is then expected to still be valid. It is also important to stress that our main theorem equally applies to mixed initial states, such as thermal states, which are relevant in present day experiments with ultracold fermions [33-36].

Returning to the specifics of quadratic Hamiltonians, the result derived here can be interpreted in yet another way: it is reminiscent of the initial state converging towards a generalized Gibbs ensemble (GGE) [10,26,37] in the sense that the initial state becomes close to a Gaussian state, which is the maximum entropy state given the second moments. Different from a real GGE, the observables $\left\{I_{\alpha}\right\}$ held fixed while maximizing entropy can be time dependent, i.e., $\rho_{G}(t)=\exp \left(\sum_{\alpha} \lambda_{\alpha} I_{\alpha}(t)\right) / \mathcal{Z}$. Here, $\left\{\lambda_{\alpha}\right\}$ are appropriately chosen Lagrange multipliers, $\mathcal{Z}$ the 
partition function, and $\left\{I_{\alpha}(t)\right\}$ the number operators of the eigenmodes of $\gamma$. However, in the case of equilibrating second moments, all relevant $I_{\alpha}$ become time independent such that our theorem constitutes a proof of a convergence to a proper GGE in these cases. The same holds true for integrable spin models that can be mapped to the type of fermionic models considered here, complementing insights on bosonic systems [6,38].

Conclusion and outlook.-In this Letter, we have established an understanding of how systems quenched to noninteracting fermionic Hamiltonians locally converge to Gaussian states. Out of equilibrium dynamics is identified as having the tendency to bring systems locally in maximum entropy states given the second moments. This holds even if the initial state was far from being a Gaussian state, e.g., a ground state of a strongly interacting model. This is achieved based on just two natural assumptions: a form of delocalizing transport in the model and exponential clustering of correlations in the initial state. Otherwise, the initial state can be completely general. It is the hope that the present work will serve as a stepping stone to gain further insights into the relaxation dynamics of more complex quantum many-body systems and the consequences of the suppression of transport in, for example, localizing systems.

We acknowledge fruitful discussions with M. Cramer, M. M. Wolf, and P. Ćwikliński. We would like to thank the EU (RAQUEL, SIQS, AQUS, IP, QUIC), the ERC (TAQ, OSYRIS, QITBOX), the Templeton Foundation, the BMBF (Q.com), the DFG (EI 519/7-1, CRC 183), the Studienstiftung des Deutschen Volkes, MPQ-ICFO, the Spanish Ministry Project FOQUS (FIS2013-46768-P), MINECO (Severo Ochoa Grant No. SEV-2015-0522), Fundació Privada Cellex, the Generalitat de Catalunya (SGR 874 and 875), ICFOnest+ (FP7-PEOPLE-2013COFUND), the EU's Marie Skłodowska-Curie Individual Fellowships programme under Grant Agreement No. 700140, and the COST Action MP1209 for support.

[1] A. Polkovnikov, K. Sengupta, A. Silva, and M. Vengalattore, Rev. Mod. Phys. 83, 863 (2011).

[2] J. Eisert, M. Friesdorf, and C. Gogolin, Nat. Phys. 11, 124 (2015).

[3] C. Gogolin and J. Eisert, Rep. Prog. Phys. 79, 056001 (2016).

[4] I. Bloch, J. Dalibard, and S. Nascimbene, Nat. Phys. 8, 267 (2012).

[5] S. Trotzky, Y.-O. Chen, A. Flesch, I. McCulloch, U. Schollwöck, J. Eisert, and I. Bloch, Nat. Phys. 8, 325 (2012).

[6] M. Cramer, C. M. Dawson, J. Eisert, and T. J. Osborne, Phys. Rev. Lett. 100, 030602 (2008).

[7] P. Reimann, Phys. Rev. Lett. 101, 190403 (2008).

[8] N. Linden, S. Popescu, A. J. Short, and A. Winter, Phys. Rev. E 79, 061103 (2009).

[9] A. J. Short and T. C. Farrelly, New J. Phys. 14, 013063 (2012).
[10] M. Rigol, V. Dunjko, V. Yurovsky, and M. Olshanii, Phys. Rev. Lett. 98, 050405 (2007).

[11] P. Reimann and M. Kastner, New J. Phys. 14, 043020 (2012).

[12] A. S. L. Malabarba, L. P. Garcia-Pintos, N. Linden, T. C. Farrelly, and A. J. Short, Phys. Rev. E 90, 012121 (2014).

[13] D. Pertot, A. Sheikhan, E. Cocchi, L. A. Miller, J. E. Bohn, M. Koschorreck, M. Köhl, and C. Kollath, Phys. Rev. Lett. 113, 170403 (2014).

[14] M. B. Hastings and T. Koma, Commun. Math. Phys. 265, 781 (2006).

[15] B. Nachtergaele and R. Sims, contribution to the XVth International Congress on Mathematical Physics New Trends in Mathematical Physics (2007).

[16] M. Kliesch, C. Gogolin, M. J. Kastoryano, A. Riera, and J. Eisert, Phys. Rev. X 4, 031019 (2014).

[17] See Supplemental Material at http://link.aps.org/ supplemental/10.1103/PhysRevLett.117.190602, for further details of the proof of the main argument and elaborates on applications in the study of spin chains, which includes Refs. [18-21].

[18] A. Kitaev, Ann. Phys. (Amsterdam) 321, 2 (2006).

[19] E. Lieb, T. Schultz, and D. Mattis, Ann. Phys. (N.Y.) 16, 407 (1961).

[20] J. H. van Lint and R. M. Wilson, A Course in Combinatorics (Cambridge University Press, Cambridge, United Kingdom, 1992).

[21] C. V. Kraus, M. M. Wolf, J. I. Cirac, and G. Giedke, Phys. Rev. A 79, 012306 (2009).

[22] E. H. Lieb and D. W. Robinson, Commun. Math. Phys. 28, 251 (1972).

[23] M. B. Hastings, Phys. Rev. Lett. 93, 126402 (2004).

[24] A. Flesch, M. Cramer, I. P. McCulloch, U. Schollwöck, and J. Eisert, Phys. Rev. A 78, 033608 (2008).

[25] P. Calabrese and J. Cardy, Phys. Rev. Lett. 96, 136801 (2006).

[26] P. Calabrese, F. H. Essler, and M. Fagotti, J. Stat. Mech. (2012) P07022.

[27] I. Peschel and V. Eisler, J. Phys. A 42, 504003 (2009).

[28] M. Fagotti and F. H. L. Essler, Phys. Rev. B 87, 245107 (2013).

[29] S. Bhattacharyya, A. Das, and S. Dasgupta, Phys. Rev. B 86, 054410 (2012).

[30] S. R. Manmana, S. Wessel, R. M. Noack, and A. Muramatsu, Phys. Rev. Lett. 98, 210405 (2007).

[31] T. V. Dudnikova, A. Komech, and H. Spohn, J. Math. Phys. (N.Y.) 44, 2596 (2003).

[32] R. L. Hudson, J. Appl. Probab. 10, 502 (1973).

[33] U. Schneider, L. Hackermuller, J. P. Ronzheimer, S. Will, S. Braun, T. Best, I. Bloch, E. Demler, S. Mandt, D. Rasch, and A. Rosch, Nat. Phys. 8, 213 (2012).

[34] M. F. Parsons, F. Huber, A. Mazurenko, C. S. Chiu, W. Setiawan, K. Wooley-Brown, S. Blatt, and M. Greiner, Phys. Rev. Lett. 114, 213002 (2015).

[35] N. Strohmaier, Y. Takasu, K. Günter, R. Jördens, M. Köhl, H. Moritz, and T. Esslinger, Phys. Rev. Lett. 99, 220601 (2007).

[36] E. Haller, J. Hudson, A. Kelly, D. A. Cotta, B. Peaudecerf, G. D. Bruce, and S. Kuhr, Nat. Phys. 11, 738 (2015).

[37] A. Lazarides, A. Das, and R. Moessner, Phys. Rev. Lett. 112, 150401 (2014).

[38] M. Cramer and J. Eisert, New J. Phys. 12, 055020 (2010). 